\title{
Challenges and Pitfalls Associated with Stem Cell Transplants for Stroke
}

\section{Carli L Roulston*}

Department of Medicine, St Vincent's Campus, University of Melbourne, Melbourne, Victoria, Australia

Although new reports suggest that the therapeutic window for thrombolysis after stroke might be extended [1], there is still a compelling need to develop treatment strategies that target the majority of patients. Nevertheless, a slow but consistent recovery is often observed across a cross section of patients during rehabilitation without pharmaceutical intervention. Studies now show that the injured brain is primed for repair processes and brain remodelling contributes to functional recovery in many people following ischaemic stroke [2]. To this end, ischaemic insults have now been shown to trigger neural progenitor cell (NPC) proliferation and stem cell migration from the subventricular zone (SVZ) of the lateral ventricle to damaged regions of the brain [3], even in patients of advanced age [4]. Despite this attempt at endogenous repair, there is little evidence to suggest that significant improvement in neurological function during rehabilitation after stroke is attributed to neuronal replacement [2]. Rather, studies have now shown that migrating NPC's remain outside the ischaemic territory, and most differentiate into astrocytes, potentially contributing to gliotic scar formation [5]. This raises the question of whether or not further recovery from stroke might be achieved if lost neurons were to be replaced.

For reasons above, development of neuro-restorative strategies to facilitate endogenous neuronal replacement presents a challenging new approach for stroke. Consequently exogenous cell-based therapies to complement endogenous repair mechanisms are currently being trialled in humans [6]. Although preclinical data supports their efficacy, little is known about the fate of these cells in the human brain and identifying factors that influence transplant survival, differentiation and integration with existing neuronal circuits in human patients is imperative for achieving translational success [7]. Establishment of the most suitable conditions in the brain to support neuronal recovery and replacement, as well as determining optimal timing for transplant success after stroke, are still key focus areas that are yet to be comprehensively addressed $[8,9]$.

The goal of cell based therapies is to support 'at risk' neurons that are still participating in neural signalling after stroke and to 'reconstruct' a lost neural circuit over time by administering a cell source that can integrate into and participate in functional neural circuitry. It is also desirable to implant and generate new neurons in addition to supporting the survival of cells already in situ. The search for the most suitable source of a restorative cell type for neural repair has been undertaken with great vigour and includes embryonic, foetal derived and non-neural adult-derived stem cells. Although survival of these cells following transplant has been documented in animal models, significant differentiation into functioning mature neurons is yet to be realised $(<0.2)$ [7,10]. A supportive microenvironment may be necessary for ensuring long term graft survival and functional integration of cells into the damaged brain. Perhaps it is this reason that early clinical trials chose not to implant sooner than 6 months after stroke to avoid the acute phase associated with toxicity and infarct expansion [11]. However recent studies suggest that this late time may not be optimal either due to extensive glial scar formation and loss of factors that are known to support cell survival [12].

During the acute phase of stroke, microglia are activated within minutes, with peak inflammatory cell infiltration detected in animal models by 7 days $[12,13]$, contributing to injury through several mechanisms. Astrogliosis is also detected between 3 and 7 days after stroke with evidence of gliotic scar formation commencing around the infarct border by 14 days, which thickens to eventually occupy the core infarct $[12,14]$. The neurovascular unit is also damaged primarily by reduced blood flow and secondarily by ensuing inflammatory processes. Vascular recovery in the form of angiogenesis is detected early after stroke and has been implicated in supporting brain regeneration, but new vascular networks are not evident until at least 14 days [12], and then later regress by 6 months if recovery is not achieved [15]. Collectively, these factors contribute to an environment that is challenging for neuronal survival, repair and replacement: timing is thus one of the most crucial factors for consideration in transplant studies. Importantly, transplantation of stem cells may need to be combined with strategies that promote a more supportive microenvironment with reduced inflammation and scar formation, plus increased vascularisation for neuronal survival.

Although capable of forming neuronal populations after transplant in animal models of neurotrauma, NPCs predominantly differentiate into astrocytes [10,16-18]. Astrogliosis in the injured brain is not a simple all-or-none phenomenon, but a finely gradated continuum of morphological changes regulated by specific signalling events that range from influence on brain preservation, to long-lasting scar formation with rearrangement of tissue structure $[14,19]$. What affect stem cell derived astrocytes might have on the human brain is unknown, however functional improvements with transplanted stem cells in pre-clinical animal models has been associated with release of growth factors that are supportive of brain remodeling. On the other hand, accentuation of the glial scar may in the long term impede brain plasticity associated with axonal elongation.

In order to facilitate direct neuronal replacement, optimization of protocols designed to pre-differentiate NPCs into a neuronal phenotype prior to transplant offer new hope. Indeed recent studies in other models of neurological disease have shown that pre-differentiating cells prior to transplant results in greater functional recovery: differentiated GABAergic human NPC's injected into the spinal cord following spinal cord injury improved long term survival of GAGAergic cells that generated glutamic acid decarboxylase, GABA, and $\beta$-III tubulin, with greater functional improvement compared to animals receiving non-

*Corresponding author: Dr Carli Roulston, Team Leader Neurotrauma, Department of Medicine, Level 4 Clinical Sciences Building, St Vincent's Hospital, 29 Regent Street, Fitzroy VIC 3065, Australia, Tel: +64 39288 3242; Fax: +64 3 9288 3505; E-mail: carlir@unimelb.edu.au

Received November 26, 2013; Accepted November 28, 2013; Published December 05, 2013

Citation: Roulston CL (2013) Challenges and Pitfalls Associated with Stem Cell Transplants for Stroke. J Neurol Neurophysiol 4: e114. doi:10.4172/21559562.1000e114

Copyright: (c) 2013 Roulston CL. This is an open-access article distributed under the terms of the Creative Commons Attribution License, which permits unrestricted use, distribution, and reproduction in any medium, provided the original author and source are credited. 
differentiated cells [16]. Additionally, transplanted pre-differentiated GABAergic neurons have also been shown to improve functional outcomes in animal models of Huntington's and Parkinson's disease $[17,18]$. Moreover, GABAergic hNPC's transplanted directly into the diseased brain were non-proliferative, demonstrating the stability of these cells and their reduced risk of teratogenic potential often associated with undifferentiated pluripotent stem cells.

Characterisation of factors that both hinder and aid brain repair is paramount to achieving successful outcomes for stem cell therapies aimed at improving and accelerating functional recovery. Timing after stroke is crucial and combined therapeutic strategies that target reduced inflammation and scar formation may offer added benefit. Equally strategies that facilitate long term re-vascularisation of the injury brain may be necessary to support long term survival of transplanted cells. Finally, optimisation of protocols designed to pre-differentiate cells into a desired neuronal phenotype prior to transplant may be a better approach for stably re-populating the damaged brain, not just for stroke but for all neurodegenerative disease.

\section{References}

1. Ma H, Parsons MW, Christensen S, Campbell BC, Churilov L, et al. (2012) A multicentre, randomized, double-blinded, placebo-controlled Phase III study to investigate EXtending the time for Thrombolysis in Emergency Neurological Deficits (EXTEND). Int J Stroke 7: 74-80.

2. Gauthier LV, Taub E, Perkins C, Ortmann M, Mark VW (2008) "Remodeling the brain: plastic structural brain changes produced by different motor therapies after stroke", Stroke 39: 1520-1525.

3. Zhang RL, Zhang GZ, Chopp M (2008) "Ischaemic stroke and neurogenesis in the subventricular zone. Neuropharm" 55: 345-352.

4. Minger SL, Ekonomou A, Carta EM, Chinoy A, Perry RH, et al. (2007) "Endogenous neurogenesis in the human brain following cerebral infarction", Regen Med 2: 69-74.

5. Garzón-Muvdi T, Quiñones-Hinojosa A (2009) "Neural stem cell niches and homing: recruitment and integration into functional tissues", ILAR J 51: 3-23.

6. Kalladka D, Muir KW (2011) Stem cell therapy in stroke: designing clinical trials. Neurochem Int 59: 367-370.
7. Leong WK, Lewis MD, Koblar SA (2013) Concise review: Preclinical studies on human cell-based therapy in rodent ischemic stroke models: where are we now after a decade? Stem Cells 31: 1040-1043.

8. Savitz SI, Chopp M, Deans R, Carmichael ST, Phinney D, et al. (2011) Stem Cell Therapy as an Emerging Paradigm for Stroke (STEPS) II. Stroke 42: 825829

9. Christie KJ, Turnley AM (2013) Regulation of endogenous neural stem/ progenitor cells for neural repair-factors that promote neurogenesis and gliogenesis in the normal and damaged brain. Front Cell Neurosci 6: 70.

10. Arien-Zakay H, Lecht S, Bercu MM, Tabakman R, Kohen R, et al. (2009) "Neuroprotection by cord blood neural progenitors involves antioxidants, neurotrophic and angiogenic factors". Exp Neurol 216: 83-94.

11. Kondziolka D, Wechsler L (2008) "Stroke repair with cell transplantation: neuronal cells, neuroprogenitor cells, and stem cells". Neurosurg.24: E13.

12. Taylor CJ, Weston RM, Dusting GJ, Carli L Roulston (2013) "NADPH oxidase and angiogenesis following endothelin-1 induced stroke in rats: role for Nox2 in brain repair". Brain Sci 3: 294-317.

13. Yenari MA, Kauppinen TM, Swanson RA (2010) "Microglial activation in stroke: therapeutic targets". Neurotherapeutics 7: 378-391.

14. Sofroniew MV, Vinters HV (2010) "Astrocytes: biology and pathology". Acta Neuropathol 119: 7-35

15. Yu SW, Friedman B, Cheng Q, Lyden PD (2007) Stroke-evoked angiogenesis results in a transient population of microvessels. J Cereb Blood Flow Metab 27: 755-763.

16. Mukhida K, Mendez I, McLeod M, Kobayashi N, Haughn C, et al. (2007) "Spinal GABAergic transplants attenuate mechanical allodynia in a rat model of neuropathic pain". Stem Cells 25: 2874-2885.

17. Mukhida K, Hong M, Miles GB, Phillips T, Baghbaderani BA, et al. (2008) "A multitarget basal ganglia dopaminergic and GABAergic transplantation strategy enhances behavioural recovery in parkinsonian rats". Brain 131: 2106-2126.

18. McLeod MC, Kobayashi NR, Sen A, Baghbaderani BA, Sadi D, et al. (2012) Transplantation of GABAergic Cells Derived from Bioreactor-Expanded Human Neural Precursor Cells Restores Motor and Cognitive Behavioural Deficits in A Rodent Model of Huntington's Disease. Cell Transplant.

19. McGraw J, Hiebert GW, Steeves JD (2001) "Modulating astrogliosis after neurotrauma". J Neurosci Res 63: 109-115. 